\title{
A FUZZY PETRI NETS QOS MODEL FOR WIRELESS AD HOC NETWORKS
}

\author{
Lyes Khoukhi ${ }^{1}$ and Soumaya Cherkaoui ${ }^{1}$ \\ ${ }^{1}$ Department of Electrical and Computer Engineering, Université de Sherbrooke J1K \\ 2R1, QC, Canada
}

\begin{abstract}
In this paper, we explore the use of Fuzzy Petri Nets for QoS support in wireless ad hoc networks. We propose a fuzzy Petri nets technique for modeling and analyzing the QoS decision making for traffic regulation. The proposed model, called FPWIM, studies the fuzzy regulation traffic rules in order to deal with the imprecise information caused by the dynamic topology of ad hoc networks. The input parameters of FPWIM are the node mobility and the delay measurement received by a node as feedback information from the MAC layer. The output parameter of FPWIM is the traffic regulation rate necessary to avoid the possible congestion in the network. Different traffic and network motilities are considered by FPWIM in order to help make an efficient QoS decision for various network conditions.
\end{abstract}

Keywords: ad hoc networks; quality of service; fuzzy Petri networks.

\section{INTRODUCTION}

As a widespread use of wireless technology, the ability of mobile wireless ad hoc networks to support real-time services with Quality of Service (QoS) has become a challenging research subject. This challenge is due essentially to the fact that the wireless topology can change rapidly in unpredictable ways or remain relatively static over long periods of time. In addition, the dynamic topology of ad hoc networks generates imprecise and uncertain information, which may complicate the task of QoS and routing protocols.

Some researches have focused on aspects such as QoS routing [7]-[8] and MAC layer [9]. Other recent researches have focused on presenting models that enable QoS support independent of the routing protocols. The most noteworthy QoS models attempting to establish comprehensive solutions for MANETs (Mobile Ad hoc Networks) are INSINGIA [2], SWAN [3], and FQMM [4]. We have proposed in [5], an intelligent QoS model with service differentiation based on neural networks in mobile ad 
hoc networks named GQOS. In [6], we have developed the FuzzyMARS model which explores the use of a fuzzy logic semi-stateless QoS approach comparatively to using neural networks. The use of fuzzy logic showed very interesting results such as the reduction of the average endto-end delay of traffic. This study aims at giving a good analytical model for using fuzzy logic for traffic regulation in MANETs.

The chosen model is fuzzy Petri Nets. The classical Petri nets [15] are not sufficient to model the dynamic topology of MANETs characterized by the uncertainty and imprecision information. It has been proven that the imprecise information can be represented efficiently by using Fuzzy Petri Nets model [10] [11] [12] [13].

The proposed fuzzy modeling scheme for traffic regulation aims to represent the dynamic adjustment of traffic transmission according to the network conditions. We called this model "FPWIM". FPWIM exploits the fuzzy concepts to model the QoS approach decision making. The representation of different fuzzy processes for decision making can be performed by formulating the production rules of these processes. Each fuzzy production rule is a set of antecedent input conditions and consequent output propositions. We proceed to construct the previous aspects (the input and output parameters) of the production rules in order to better represent and understand the process of traffic regulation in wireless ad hoc networks. The traffic regulation used to avoid the congestion depends on the traffic state and the dynamic topology of the network. The input parameters of FPWIM are the node mobility and the delaymeasurement. This later parameter is received by a node as feedback information from the MAC layer; it represents the time taken by packet to reach the destination. The delay measurement parameter can give information about the status of a network in terms of congestion. A big value of this parameter signifies that congestion may have appeared in the network. Therefore, the process of traffic regulation should be started. The amount of this regulation represents the output parameter of FPWIM. The fuzzy Petri nets tool is used for its efficiency and flexibility over other modeling tools in the aim of better modeling and representation the process of traffic regulation.

This paper is organized as follows: in Section II, we describe the fuzzy Petri nets tool. Section III illustrates the fuzzy regulation traffic rules used by FPWIM. The fuzzy Petri nets model for traffic regulation is shown in Section IV. Finally, Section IV concludes the paper.

\section{FUZZY PETRI NETS}

Classical Petri Nets [15] do not have sufficient capacity to model the uncertainty in systems [14] [18]. This limitation of Petri nets has encouraged researchers to extend the exiting models by using the fuzzy reasoning theory [10] [11] [13]. The combination of Petri nets models and 
fuzzy theory has given rise to a new modeling tool called Fuzzy Petri Nets (FPN). FPN formalism has been widely applied in several applications such as, fuzzy reasoning systems [16], robotics systems [12], and real-time control system [14], etc.

In what follows, we give a brief description about the FPN modeling tool [10] [12]. Let consider FPN $=(P N, C N D, M F, F S R, F M)$.

The tuple $P N=(P, T, A, F W, F H)$ is called Petri nets if: $(P, T, A)$ is a finite net, where [14]:

$P=\left\{P_{1}, P_{2}, \ldots, P_{n}\right\}$ is a finite non-empty set of places,

$\mathrm{T}=\left\{\mathrm{T}_{1}, \mathrm{~T}_{2}, \ldots, \mathrm{T}_{\mathrm{n}}\right\}$ is a finite non-empty set of transitions,

$A \subseteq(P \times T) \cup(T \times P)$ is a finite set of arcs between the places and transitions or vice versa.

$\mathrm{FW}: \mathrm{A} \rightarrow \mathrm{N}^{+}$represents a weighting function that associates with each arc of $\mathrm{PN}$ a non-negative integer of $\mathrm{N}^{+}$.

$F H \subset(P \times T)$ : represents an inhibition function that associates a place $P_{i} \in P$ contained in $\mathrm{FH}\left(\mathrm{T}_{\mathrm{j}}\right)$ to a transition $\mathrm{T}_{\mathrm{j}}$ itself.

a) $\mathrm{CND}=\left\{\mathrm{cd}_{1}, \mathrm{~cd}_{2}, \ldots, \mathrm{cdn}\right\}$ represents a set of conditions that will be mapped into the set $\mathrm{P}$; each $c d_{i} \in C N D$ is considered as one input to the place $P_{i} \in P$. A condition cd $_{i}$ takes the form of "X is $Z$ ", which means a combination between the fuzzy set $Z$ and the attribute $X$ of the condition. For instance, in the condition "the delay measurement is small", the attribute " $\mathrm{X}=$ delay measurement" is associated to the fuzzy set " $Z=$ small", but other fuzzy sets can also be considered (e.g. " $Z=$ medium", " $Z=$ large", etc.).

b) Consider MF: $u_{Z}(x) \rightarrow[0,1]$, a membership function which maps the elements of $X$ (as defined in $b$.) into the values of the range $[0,1]$. These values represent the membership degree in the fuzzy set $Z$. The element $x$ belonging to $X$ represents the input parameter of the condition " $\mathrm{X}$ is $\mathrm{Z}$ ", and $u_{z}(x)$ measures the degree of truth of this condition. Note that the composition of membership function degrees of the required conditions is performed by fuzzy operators such as MNN/MAX.

c) Let consider the following rule $R_{i}$ : " $R_{i}$ : if $x_{1}$ is $z_{1}$ and /or $x_{2}$ is $z_{2}$ then $A$ is $B$ ". The firing strength function of rule $R_{i}\left(F S R_{i}\right)$ represents the strength of belief in $R_{i}$. The conclusion of $R_{i}$ (modeled by $C S R_{i}$ ) can take one of the following forms:

$$
\begin{aligned}
& C S R=\operatorname{MIN}\left(u_{x 1}\left(x_{1}\right), u_{z 2}\left(x_{2}\right)\right)=u_{x 1}\left(x_{1}\right) \wedge u_{z 2}\left(x_{2}\right) \\
& C S R_{r}=\operatorname{MAX}\left(u_{z 1}\left(x_{1}\right), u_{z 2}\left(x_{2}\right)\right)=u_{z 1}\left(x_{1}\right) \vee u_{z 2}\left(x_{2}\right)
\end{aligned}
$$

d) SWR is the selected wining rule $R_{L}$ among the n-rules $R_{1}, R_{2}, \ldots, R_{n}$. SWR is the rule which has the highest degree of truth. Let FSR $R_{L}$ be the corresponding firing strength of $R_{L}$, then the selected rule SWR is given as follows:

$$
S W R=M A X\left(F S R_{1}, F S R_{2}, \ldots, F S R_{n}\right)
$$

e) The marking task in FPN illustrates the satisfaction of events occurred during the performance of fuzzy rules. This marking function called "fuzzy marking" (FM) distributes the tokens over the places of the 
nets. A token is the primitive concept used in classical Petri nets for the definition of their execution.

f) The sequence $\delta=\left\langle T_{1}, T_{2}, \ldots, T_{n}\right\rangle$ is said to be reachable from a fuzzy marking $\mathrm{FM}_{1}$, if $T_{i} \in T$ is a firable from $\mathrm{FM}_{1-1} \in \mathrm{FM}$ and leads to $\mathrm{FM}_{\mathrm{i}+1} \in \mathrm{FM}$, for all transitions $T_{i} \in \delta$. The firing of transition $T_{i} \in T$ (Figure 1) is performed in two steps: a) $T_{i}$ removes tokens and then, b) $T_{i}$ places tokens.

\section{FUZZY REGULATION TRAFFIC RULES USAGE}

Most of fuzzy systems use the following form for modeling [1] [19] [17]: Rule R: if $\mathrm{Ip}_{1}$ is $\mathrm{A} A N D \mathrm{Ip}_{2}$ is $B$ then Op is $\mathrm{C}$

Where:

- $I p_{1}$ and $I p_{2}$ are the input parameters,

- Op is an output parameter,

- A, B, and C are fuzzy sets,

- AND represent fuzzy operator,

- The fuzzy conditions of rule $R$ are "I $p_{1}$ is A", and " $I p_{2}$ is B".

The construction of the above aspects (inputs, outputs, and fuzzy sets) for performing the traffic regulation to avoid the possible congestion depends on the traffic state and the dynamic topology of wireless ad

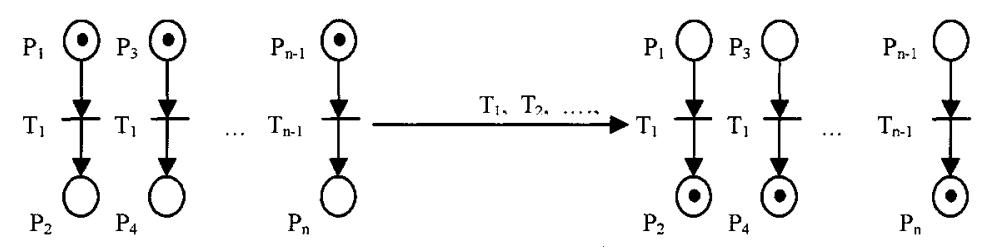

Figure 1. The transitions firing in FPN

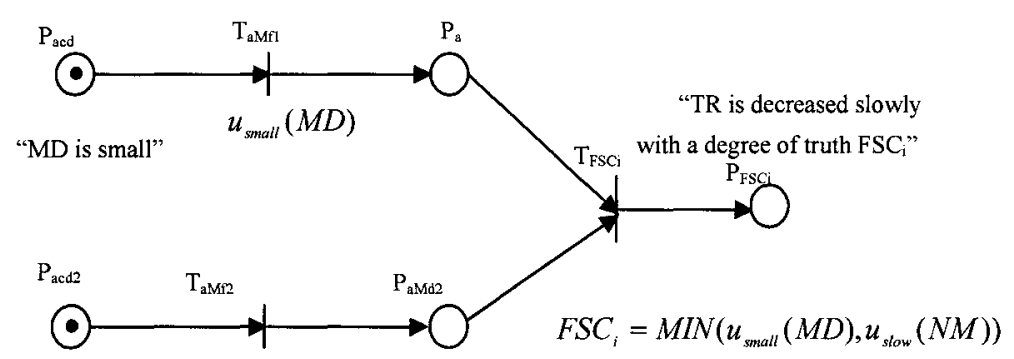

"NM is slow" $\quad u_{\text {slow }}(N M)$

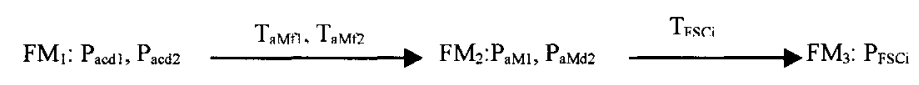

Figure 2. The modeling of fuzzy rules structure and its dynamic behaviour 
hoc networks. Thus, the previous fuzzy aspects can take various values:

- The first input parameter: is represented by the Delay-Measurement (DM) at a mobile node. DM can be either small or large.

- The second input parameter: is represented by the Node Mobility (NM). NM can either be slow or medium (note that "fast node mobility" is included in the case of "medium node mobility").

- The output parameter: is represented by the Traffic regulation rate (TR). TR can either be decreased or increased (slowly or largely).

The aim is to help to establish production rules that make an efficient QoS decision. In the following, we explain the proposed fuzzy tool for the QoS decision making. Let consider the following fuzzy rule $R_{L}$ :

Rule $R_{L}$ : if DM is small and NM is slow, then TR is increased largely.

$\mathrm{R}_{\mathrm{L}}$ takes into consideration the input parameter of the feedback delaymeasurement DM and the node mobility NM. The traffic regulation rate TR represents the output parameter. Figure 2 illustrates FPN that models the dynamic aspect of the fuzzy rule $R_{L}$.

- $\mathrm{P}_{\text {acdl }}$ : models the antecedent condition $1\left(\operatorname{acd}_{1}\right)$ of $\mathrm{R}_{L}$; $\operatorname{acd}_{1}=$ "DM is small".

- $P_{a c d 2}$ : models the antecedent condition $2\left(\operatorname{acd}_{2}\right)$ of $R_{L} ; \operatorname{acd}_{2}=$ "NM is slow".

- $\mathrm{T}_{\mathrm{aMf1}}$ : models the membership function of the antecedent condition 1 ; $T_{\text {aMf } 1=} u_{\text {small }}(D M)$.

- $\mathrm{T}_{\mathrm{amf2}}$ : models the membership function of the antecedent condition 2; $T_{\text {aMf } 2}=u_{\text {slow }}(N M)$.

- $\mathrm{P}_{\mathrm{aMd1}}$ : models the membership degree value of the condition 1 of a rule $R_{L}$. This value determines the satisfaction degree of the DM input parameter to the fuzzy set "small".

- $\mathrm{P}_{\mathrm{AMD} 2}$ : models the membership degree value of the condition 2 of a rule $R_{L}$. This value determines the satisfaction degree of the NM input parameter to the fuzzy set "slow".

- T TSCL: models the operation of minimum composition "MIN" between the antecedent conditions (e.g. condition 1 and condition 2) of a rule $R_{L}$. The firing strength of $R_{L}$ is represented by the MIN operation: $M I N\left(u_{\text {small }}(D M), u_{\text {slow }}(N M)\right)$.

- $P_{\mathrm{FSCL}}$ : models the value of the firing strength of $\mathrm{R}_{\mathrm{L}}$. This value defines the degree of truth of the output proposition "TR is increased largely".

\section{FUZZY PETRI NETS MODEL FOR TRAFFIC REGULATION}

We consider the following rules:

$R_{1}$ : if DM is small and NM is slow then TR is increased largely,

$R_{2}$ : if DM is small and NM is medium then TR is increased,

$R_{3}$ : if DM is large and NM is slow then TR is decreased, 
$R_{4}$ : if DM is large and NM is medium then TR is decreased largely.

- Input parameters: The input parameter of the first antecedent condition of the rules $R_{1}, R_{2}, R_{3}$, and $R_{4}$ is the delay measurement $D M$. The input parameter of the second antecedent condition of the rules $R_{1}, R_{2}, R_{3}$, and $R_{4}$ is the node mobility NM.

- Fuzzy sets: The fuzzy set of the antecedent conditions of the defined rules $R_{1}, R_{2}, R_{3}$, and $R_{4}$ are: small, large, slow, and medium.

- Antecedent conditions $\left(\mathrm{acd}_{\mathrm{i}}\right)$ : The first antecedent condition $\left(\mathrm{acd}_{1}\right)$ in the rules $R_{1}, R_{2}, R_{3}$, and $R_{4}$ is: acd1: DM is small; acd2: DM is large. The second antecedent condition $\left(\operatorname{acd}_{2}\right)$ in the rules $R_{1}, R_{2}, R_{3}$, and $\mathrm{R}_{4}$ is: acd1: NM is slow; acd2: NM is medium.

- Output parameters: The output parameter of the rules $R_{1}, R_{2}, R_{3}$, and $\mathrm{R}_{4}$ is the traffic regulation rate $T R$.

- The decisions making of the rules $R_{1}, R_{2}, R_{3}$, and $R_{4}$ are: increased largely, increased, decreased, decreased largely,

- The fuzzy logic operator used by the rules $R_{1}, R_{2}, R_{3}$, and $R_{4}$ is AND. The fuzzy operator "AND" is used to combine the two antecedent conditions of each rule using the MIN function. This provides the firing strength value for each rule. After that, MAX composition function is used to combine all firing strength values of the defined rules $R_{1}, R_{2}, R_{3}$, and $R_{4}$ in the aim of determining the highest one that will be the selected wining rule. Figure 3 shows the fuzzy logic scheme for decision making of rules $R_{1}, R_{2}, R_{3}$, and $R_{4}$.

In what follows, we illustrate the steps of the proposed FPN model.

a) Enter the input parameters into the places and transitions:

- $\mathrm{P}_{\mathrm{IP}}=\left\{\mathrm{P}_{\mathrm{IP} 1}, \mathrm{P}_{\mathrm{IP} 2}, \ldots ., \mathrm{P}_{\mathrm{IP} n}\right\}$ is a set of places representing the input parameters. In the Figure 4, $P_{1}$ and $P_{2}$ represent respectively, the first (e.g. delay measurement DM) and second (e.g. node mobility NM) antecedent condition of the rules $R_{1}, R_{2}, R_{3}$, and $R_{4}$.

- $T_{I P}=\left\{T_{I P 1}, T_{I P 2}, \ldots ., T_{I P n}\right\}$ represents a set of input parameter transitions. The transitions $T_{I P 1}$ and $T_{I P 2}$ illustrated in Figure 4 are used to distribute respectively, the input parameters "DM" and "NM" for making the first and second antecedent conditions of the defined rules $\mathbf{R}_{1}, \mathbf{R}_{2}, \mathbf{R}_{3}$, and $\mathbf{R}_{4}$.

b) Represent the antecedent conditions, and compute the membership function for each condition.

- $P_{\text {acd }}=\left\{P_{\text {acd1 }}, P_{\text {acd2 }}, \ldots ., P_{\text {acdn }}\right\}$ is a set of places that represent the antecedent conditions. $P_{\text {acd } 1}$ and $P_{a c d 2}$ in the model presented in Figure 4 describe respectively, the antecedent conditions " $a \mathrm{~cd}_{1}$ " and "acd ${ }_{2}$ ".

- $T_{a M f}=\left\{T_{a M f l}, T_{a M f 2}, \ldots . ., T_{a M f n}\right\}$ is a set of transitions that represent the antecedent membership functions. $\mathrm{T}_{\mathrm{aMfl}}, \mathrm{T}_{\mathrm{aMf} 2}, \mathrm{~T}_{\mathrm{aMf}}, \mathrm{T}_{\mathrm{aMf} 4}$ observed in Figure 4 represent the membership functions of respectively, $u_{\text {small }}(D M), u_{\text {large }}(D M), u_{\text {slow }}(N M), u_{\text {medium }}(N M)$.

- $\mathrm{P}_{\mathrm{aMd}}=\left\{\mathrm{P}_{\mathrm{aMdl}}, \mathrm{P}_{\mathrm{aMd} 2}, \ldots, \mathrm{P}_{\mathrm{aMdn}}\right\}$ is a set of places that represent the antecedent membership degrees. The values of the place $\mathrm{P}_{\mathrm{amdl}}$ indi- 


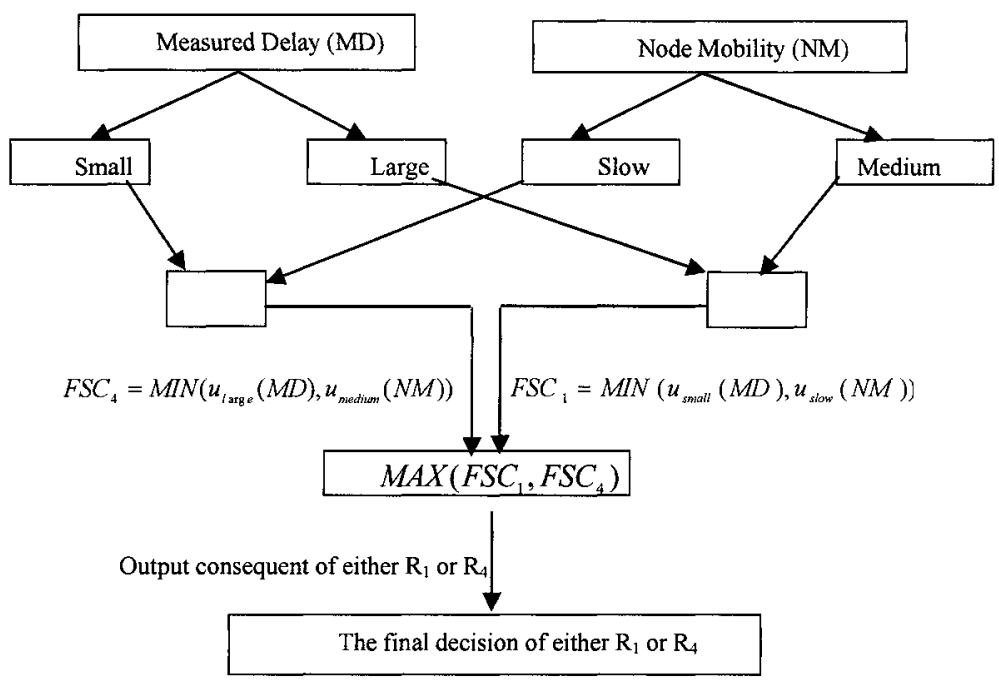

\section{Decision making algorithm:}

- Phase 1: enter the input parameters of the rules $R_{t}, R_{2}, R_{3}$, and $R_{4}$.

- Phase 2: calculate the degree of truth of the antecedent conditions.

- Phase 3: apply the operation of minimum composition (MIN) with the fuzzy operator $A N D / O R$ in order to generate the firing strength value for each rule $R_{1}, R_{2}, R_{3}$, and $R_{4}$.

- Phase 4: apply the operation of maximum composition to select the wining rule among the rules $R_{1}, R_{2}, R_{3}$, and $R_{4}$.

- Phase 5: generate the output consequent of the selected wining rule.

Figure 3. The fuzzy decision making mechanism of FPWIM

cates the degree of satisfaction of the input parameter DM to the fuzzy set "small".

c) Compute the firing strength of conditions

$-\mathrm{T}_{\mathrm{FSC}}=\left\{\mathrm{T}_{\mathrm{FSC} 1}, \mathrm{~T}_{\mathrm{FSC} 2}, \ldots, \mathrm{T}_{\mathrm{FSCn}}\right\}$ represent a set of transitions that model firing strength conditions. For instance, the transition $\mathrm{T}_{\mathrm{FSC} \text { 1 }}$ shown in Figure 4 performs the operation of minimum composition (MIN) on the antecedent conditions of the rule $\mathbf{R}_{1}: M I N\left(u_{\text {small }}(M D), u_{\text {slow }}(N M)\right)$. Note that the fuzzy operator AND is integrated with the MIN operation to combine the first and second conditions of $R_{1}$.

- $\mathrm{P}_{\mathrm{FSC}}=\left\{\mathrm{P}_{\mathrm{FSCl}}, \mathrm{P}_{\mathrm{FSC} 2}, \ldots ., \mathrm{P}_{\mathrm{FSCn}}\right\}$ is a set of places that represent the firing strength. $\mathrm{P}_{\mathrm{FSC}}$ tokens are proportional to the number of antecedent conditions of a rule $R_{i}$. This number is shown by the label illustrated between the transitions $\mathrm{T}_{\mathrm{amfi}}$ and the place $\mathrm{P}_{\mathrm{amdi}}$. The construction of the antecedent conditions of a rule $R_{i}$ is performed by firing a transition $T_{\mathrm{FSCi}}$. The inhibitor arc designed between a place $\mathrm{P}_{\mathrm{FSCi}}$ and $\mathrm{T}_{\mathrm{FSCi}}$ is useful to note that $\mathrm{T}_{\mathrm{FSCi}}$ should fire one time. 


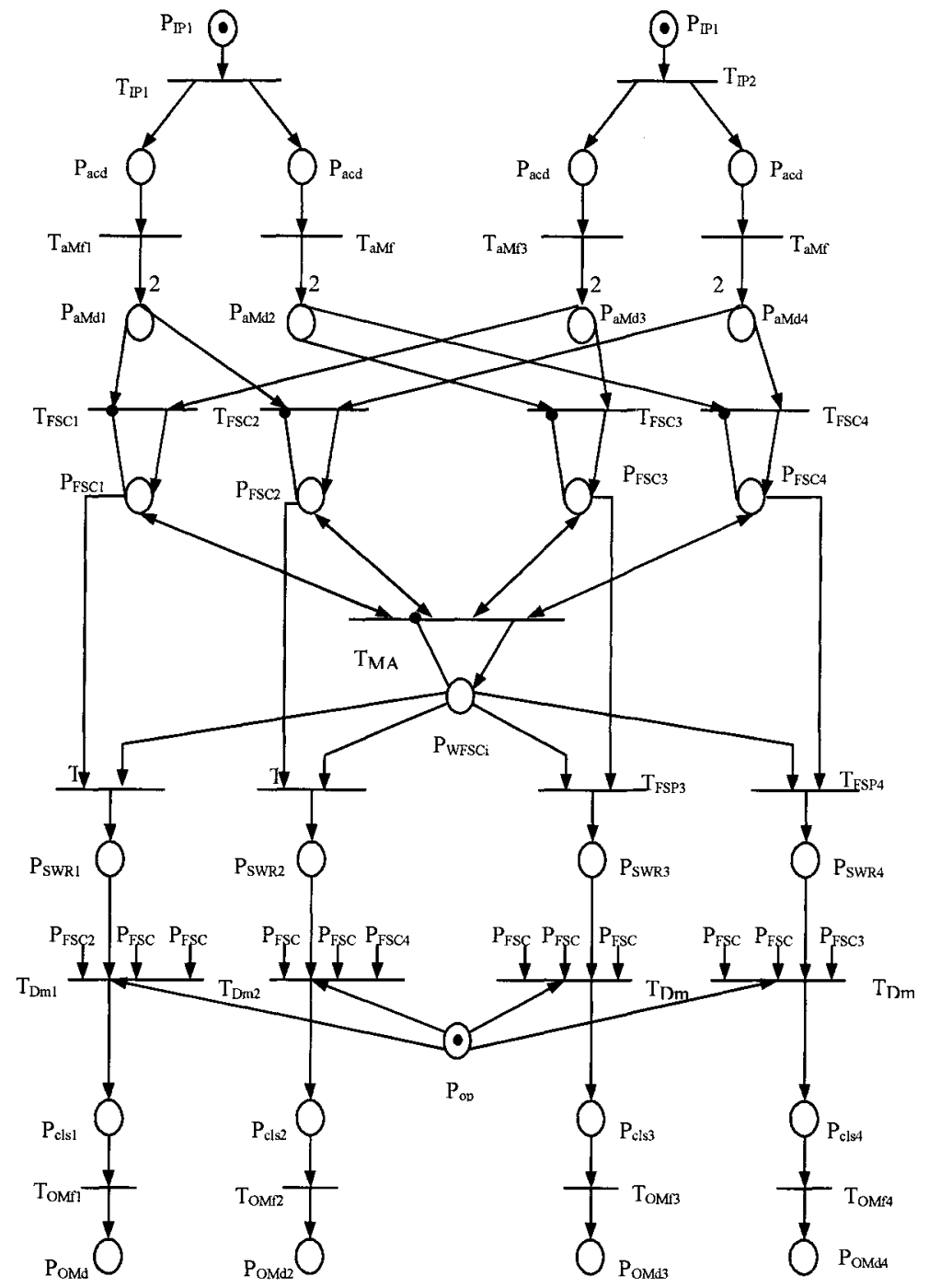

Figure 4. FPWIM model

d) Determine the selected wining rule among the activated rules:

- $\mathrm{T}_{\mathrm{FMAX}}=\mathrm{MAX}\left\{\mathrm{P}_{\mathrm{FSC} 1}, \mathrm{P}_{\mathrm{FSC} 2}, \ldots, \mathrm{P}_{\mathrm{FSCn}}\right\}$ is a transition that models the maximum composition operation (MAX) for the defined rules. The firing strength value of a rule $R_{i}$ is stored in the place $P_{\text {FSCi. }}$.

- $P_{\mathrm{WFSC}}$ represents the firing strengh condition $\mathrm{FSC}_{\mathrm{i}}$ of the selected wining rule $R_{i}$. The later rule is determined as in the following step.

- $\mathrm{T}_{\mathrm{FSP}}=\left\{\mathrm{T}_{\mathrm{FSP} 1}, \mathrm{~T}_{\mathrm{FSP} 2}, \ldots ., \mathrm{T}_{\mathrm{FSPn}}\right\}$ is a set of transitions that model the firing strength comparison. For instance, the transition $T_{F S P 3}$ is useful 
to make a comparison between $\mathrm{FSC}_{3}$ of the rule $\mathrm{R}_{3}$ and the selected wining firing strength $\mathrm{WFSC}_{\mathrm{i}}$.

- $\mathrm{P}_{\mathrm{SWR}}=\left\{\mathrm{P}_{\mathrm{SWR} 1}, \mathrm{P}_{\mathrm{SWR} 2}, \ldots, \mathrm{P}_{\mathrm{SWRn}}\right\}$ is a set of places that models the selected wining rules. The rule $R_{\mathrm{i}}$ is selected to be fired if the place $P_{\text {SWRi }}$ contains a token.

e) The conclusion of the selected rules:

- $T_{\mathrm{Dm}}=\left\{\mathrm{T}_{\mathrm{Dm} 1}, \mathrm{~T}_{\mathrm{Dm} 2}, \ldots, \mathrm{T}_{\mathrm{Dmn}}\right\}$ is a set of transitions that represent the decision of the selected rule. $T_{\mathrm{Dmi}}$ deletes the firing strength values of other rules in order to fire only the selected rule $\mathrm{R}_{\mathrm{i}}$.

- $P_{o p}$ is a place that models the output parameter. As shown in Figure 4, the place $P_{o p}$ represents the traffic regulation rate TR.

- $P_{\text {cls }}=\left\{P_{\text {cls } 1}, P_{\text {cls } 2}, \ldots, P_{c l s n}\right\}$ models a set of places that describe the different decisions of the defined rules. The places $\mathrm{P}_{\mathrm{cls} l}, \mathrm{P}_{\mathrm{cls} 2}, \mathrm{P}_{\mathrm{cls} 3}$, and $\mathrm{P}_{\text {cls }}$ illustrate the following conclusions respectively, "increased largely", "increased", "decreased", and "decreased largely". Only one place among all places will contain a token which represent the conclusion of the selected wining rule. For instance, the conclusion of the selected rule $R_{1}$ is "increased largely" if $T_{D m 1}$ transfers a token from the place $P_{\text {SWR } 1}$ to the place $P_{\text {cls } 1}$.

- $T_{\text {OMf }}=\left\{T_{\text {OMf1 }}, T_{\text {OMf2 }}, \ldots ., T_{O M f n}\right\}$ is a set of transitions that represent the output membership functions. $\mathrm{T}_{\mathrm{OMf1}}, \mathrm{T}_{\mathrm{OMf2}}, \mathrm{T}_{\mathrm{OMf3}}$, and $\mathrm{T}_{\mathrm{OMf4}}$ represent the calculation performed by the used fuzzy method to compute the membership degree of respectively,

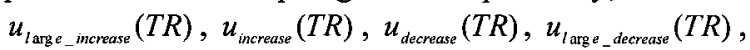

- $\mathrm{P}_{\mathrm{OMd}}=\left\{\mathrm{P}_{\mathrm{OMdl}}, \mathrm{P}_{\mathrm{OMd} 2}, \ldots ., \mathrm{P}_{\mathrm{OMdn}}\right\}$ is a set of places that represent output membership degree. The places $\mathrm{P}_{\mathrm{OMd1}}, \mathrm{P}_{\mathrm{OMd} 2}, \mathrm{P}_{\mathrm{OMd} 3}$, and $\mathrm{P}_{\mathrm{OMd}}$ indicate that the output parameters of "TR is increased", "TR is increased largely", "TR is decreased", and "TR is decreased largely" are satisfied with the following membership degree, $u_{\text {large _moreane }}(T R)$, $u_{\text {increase }}(T R), u_{\text {decrasese }}(T R), u_{\text {lare__derease }}(T R)$, respectively.

\section{CONCLUSION}

In this paper, we proposed FPWIM which is a fuzzy Petri nets technique for modeling and analyzing the QoS decision making for traffic regulation in wireless ad hoc networks. We examined the fuzzy production rules used for traffic regulation process by identifying the different parameters of each rule. The input parameters of FPWIM rules are the node mobility and the delay measurement received by a node as feedback information from the MAC layer. The output parameter of FPWIM rules is the traffic regulation rate required for reducing the possible congestion occurred in the network. The established fuzzy production rules will help deal with changing network situations in terms of mobility and congestion. This allows making an efficient QoS decision for different variable network conditions. 


\section{REFERENCES}

1. Zadeh, L. A., Knowledge representation in fuzzy logic, IEEE Transaction knowledge Data Engineering, 1, 89-100, 1989.

2. Lee, S.-B., Ahn, G.-S., Zhang, X., and Campbell, A.T., INSIGNIA: An IP-Based Quality of Service Framework for Mobile Ad Hoc Networks, Journal of Parallel and Distributed Computing, special issue on wireless and mobile computing and communication, vol. 60, no. 4, pp. 374-406, Apr. 2000.

3. Ahn, G.H., Campbell, A. T., Veres, A., and Sun, L. H., SWAN: Service Differentiation in Stateless Wireless Ad Hoc Networks, IEEE INFOCOM 2002.

4. Xiao, H., Seah, W. K.G., Lo, A., and Chaing, K., Flexible QoS Model for Mobile Ad-hoc Networks, In the Proceedings of IEEE Vehicular Technology Conference, vol. 1, pp 445-449, Tokyo, May 2000.

5. Khoukhi, L., Cherkaoui, S., A Quality of Service Approach Based on Neural Networks for Mobile Ad hoc Networks, IEEE-IFIP International Conference on Wireless and Optical Communications Networks, WOCN 2005, Dubai, UAE, Mar. 2005.

6. Khoukhi, L., Cherkaoui, S., FuzzyMARS, A Fuzzy Logic Approach with Service Differentiation for Wireless Ad hoc Networks, IEEE International Conference on Wireless Networks, Communications, and Mobile Computing WirelessCom2005, June 13-16, 2005.

7. Khoukhi, L., Cherkaoui, S., Flexible QoS Routing Protocol for Mobile Ad Hoc Networks, In the Proceedings of the 11th IEEE International Conference on Telecommunication (ICT2004), Brazil, Aug. 2004.

8. Lin, C. R., and Liu, J.-S., QoS Routing in Ad Hoc Wireless Networks, IEEE Journal on Selected Areas in Communication, vol. 17, no. 8, 1426-1438, 1999.

9. Lin, C.-R.: On-Demand QoS Routing in Multihop Mobile Networks, IEEE INFOCOM 2001, pp. 1735-1744, April 2001.

10. Ashon, S.I., Petri net models of fuzzy neural networks, IEEE Transaction on System, Man, and Cybernetics, 25, 926-932, 1995.

11. Chen, S.-M., Ke, J.-S., and Chang, J.-F., knowledge representation using fuzzy Petri nets, IEEE Transaction on Knowledge Data Engineering, 2, 311-319, 1990.

12. Cao, T., Sanderson, A.C., Variable reasoning and analysis about uncertainty with fuzzy Petri nets, In the proceeding of 14 th International conference on application and theory of Petri nets, Troy, NY, August, pp. 126-175.

13. Looney, G., Fuzzy Petri nets for rule-based decision making, IEEE Transaction on System, Man, and Cybernetics, 18, 178-183, 1998.

14. Murata, T., Suzuki, T., and Shatz, S. M., Fuzzy-timing high-level Petri net model of a real-time network protocol, In the proceeding of ITC-CSCC 96, Seoul, Korea, July 15-17, pp. 1170-1173.

15. Dwyer, M. B., and Clarke, L. A., A compact Petri net representation and its implication for analysis, IEEE Transaction Software Engineering, 22, 794-811, 1996.

16. Chaudhury, A., Marinescu, D. C., and Whinston, A., Net-based computational models of knowledge processing systems, IEEE Expert, 8, 79-86, 1993.

17. Eshera, M. A., and Barash, S. C., Parallel rule-based fuzzy inference on meshconnected systolic array, IEEE Expert, winter, 4, 17-35, 1989.

18. Murata, T., Temporal uncertainty and fuzzy-timing high level Petri nets, In the proceeding of $17^{\text {th }}$ International Conference of Application and Theory of PNS, Osaka, Japan, June 26, pp. 11-28, 1996.

19. Polat, F, and Guvenir, H., UVT: A unification-based tool for knowledge base verification, IEEE Expert, 8, 69-75, 1993. 